\title{
Assessment of malaria as a public health problem in and around Arjo Didhessa Sugar Cane Plantation area, Western Ethiopia
}

Mebrate Dufera ( $\square$ mebratedufera@gmail.com )

Wollega University

Regea Debsu

Wollega University

Gemechu Tiruneh

University of Wollongong

Research article

Keywords: Arjo Didhessa, malaria, malaria risk factors

Posted Date: October 3rd, 2019

DOI: https://doi.org/10.21203/rs.2.15519/v1

License: (c) (i) This work is licensed under a Creative Commons Attribution 4.0 International License.

Read Full License

Version of Record: A version of this preprint was published at BMC Public Health on May 12th, 2020. See the published version at https://doi.org/10.1186/s12889-020-08784-5. 


\section{Abstract}

Background: Although much progress has been made in reducing malaria morbidity and mortality worldwide in the last decade, malaria still remains the third leading cause of death and still considered as major public health problem.

Objectives : The main objective of this study was to assess malaria as a public health problem in and around sugar cane plantation area of Arjo Didhessa sugar factory, Western Ethiopia.

Methods: A community based cross sectional study supplemented with clinical retrospective data, which included 452 study subjects was employed and the study period extended from May 2016 up to November of 2017. A standardized questionnaire was used to assess malaria risk factors and blood samples were received from all study participants and further subjected to Giemsa staining for determination of malaria prevalence. Data was analyzed by SPSS version 20.Malaria risk factors were identified by multivariate logistic regression at significance level of $\mathrm{P}<0.05$.

Results: The overall malaria prevalence was 3.1\%; Plasmodium vivax being the main type of malaria parasite. Overnight outdoor sleeping and improper utilization of mosquito bed nets were found to be statistically significant as malaria risk factors in the community. In the retrospective studies of five years, the peak malaria cases (13.84\%) were reported in 2013 and less cases (1.24\%) in 2017.

Conclusion: The prevalence of malaria observed in the area is still higher as compared to national prevalence of malaria. Therefore; we recommend further strengthening of malaria prevention and control strategies. Additionally, educative training opportunities must be provided for workers in the plantation area on malaria prevention and control.

Key words : Arjo Didhessa, malaria, malaria risk factors

\section{Background}

Malaria is a heamoparasitic disease caused by obligate intracellular protozoan parasites of plasmodium species which are transmitted by infected female anopheline mosquito. There are five species of plasmodium parasites which infect humans and causes malaria, of which Plasmodium falciparum and Plasmodium vivax have a cosmopolitan distribution which holds true for the case of Ethiopia as well [1].

In the last decade, although much progress has been made in reducing malaria morbidity and mortality, next to HIV/AIDS and TB malaria still remains the third leading cause of death. Malaria still considered as major public health problem, causing considerable amount of mortality, morbidity and economic burden affecting all part of the community to which the problem is aggravated in sub Saharan African countries [2].

World Malaria Report 2018 [3] indicated that, after an unprecedented period of success in global malaria control, progress has stalled. Data from 2015-2017 highlight that no significant progress in reducing 
global malaria cases was made in this period. There were an estimated 219 million cases and 435000 related deaths in 2017.

Ethiopia bears a particularly large burden, having among the highest rates of transmission worldwide. According to national malaria indicator survey taken in 2015 [4], malaria parasite prevalence by microscopy was 0.5 percent among all age groups residing in malarious areas and a total of 2,174,707 malaria cases were detected and $(63.7 \%)$ of these cases were Plasmodium falciparum. More than half $(60 \%)$ of Ethiopia's population lives in malarious areas, and 68 percent of the country's land mass is favorable for malaria transmission [5]. Transmission takes place all year round with a seasonal peak extending from June to September which is considered as a major transmission season in the country. Malaria transmission tends to be highly heterogeneous geo-spatially within each year as well as between years. Additionally, malaria in Ethiopia is characterized by widespread epidemics occurring every five to eight years [6].

Many factors affect the dynamics of malaria transmission and infection, ranging from social to natural. Rainfall and temperature can be considered as the major natural risk factors affecting the life cycle of malaria parasite and mosquito breeding. Relative humidity plays a role in the life span of the mosquito. In the presence of high relative humidity values, the malaria parasite would complete the necessary life cycle in order to increase transmission of the infection to humans. Most districts in Ethiopia show favorable climatic conditions for development and transmission of malaria. Studies on prevalence of malaria are important not only to assess the problem of malaria in a given region, but also to analyze the effectiveness of strategies for primary and secondary prevention as well as its quality and impact.

About half of the total population living between altitudes of 1,500 and 2,500 m above sea level is at risk of malaria and the areas experience epidemics in Ethiopia [7]. Some studies [5] from high-altitude areas identified age, nearness of houses to breeding places, sharing of houses with animals, presence of windows and open eaves as malaria risk factors. In addition to this, malaria is associated with environmental factors such as altitude, rainfall, and temperature. Thus, malaria interventions target both households and environment.

Efforts to control malaria include environmental management, insecticide sprays and use of insecticidetreated nets (ITNs) [8].Since the development of the Global Malaria Control Strategy by the World Health Organization in 1992, emphasis in malaria control has shifted from vector eradication to increased case detection and treatment of malaria [6].The main malaria control strategies in Ethiopia include: early diagnosis and prompt treatment, selective vector control, epidemic management and control, environmental management and personal protection through the use of insecticide-treated bed nets [8]. Unstable malaria transmission patterns make Ethiopia prone to focal and multifocal epidemics that have on occasion caused catastrophic public health emergencies. Malaria is seasonal in most parts of Ethiopia, with variable transmission and prevalence patterns affected by the large diversity in altitude, rainfall, and population movement. Control of malaria is hinged on key global strategies, which include prompt and effective case management, intermittent preventive treatment (IPT) of malaria in pregnancy 
and integrated vector management (IVM) comprising the use of insecticide-treated nets (ITN), indoor residual spraying (IRS), and environmental management [2].

According to retrospective trend analysis of malaria cases done in Ataye District Hospital [9], a total of 31,810 blood films were examined from malaria suspected patients from January 2013 to December 2017. Of the examined blood films, 2,670 (8.4\%) were microscopically confirmed malaria cases. In 2016, higher number $(8,066)$ of malaria suspected patients were examined and $863(10.7 \%)$ of them were become microscopically confirmed cases. On the other hand, out of 6,172 malaria suspected patients, the least number of cases, 358 (5.8\%) were recorded in 2017. Generally, malaria cases showed an increment from 2013 to 2016 where as there was a decrease in malaria cases in 2017. A ten year retrospective malaria trend analysis conducted in Sibu-Sire, western Ethiopia from $2004-2013$ [10] demonstrated that, among a total of 30,070 blood films requested for malaria diagnosis, 6,036 (20.07\%) microscopically confirmed malaria cases were reported with mean malaria cases of 603.6. In this area mal aria was reported in all years with lowest (1.6\%) malaria cases reported in 2008 and the highest (31.2\%) in 2004, followed by 2010,2005 with the prevalence rate of $13.7 \%$ and $13 \%$, respectively. Furthermore, malaria aroused in all months of the year with different fluctuation rate in which, the highest peak was in June with a prevalence rate of $18.9 \%$, followed by May, November, and July with a prevalence rate of $13.3 \%$, $13.2 \%$, and $11.2 \%$, respectively [10].

To our knowledge level, the present study is the first community based malaria survey in the vicinity and can be considered as a baseline survey which would be helpful in providing information and fill the knowledge gap regarding malaria prevalence, predictors of malaria prevalence and the fluctuating trend of malaria observed over the years around the area. Thus this study was designed to assess the prevalence of malaria and its associated factors in and around Arjo Didhessa sugar factory, Western, Ethiopia.

\section{Methods}

\section{Study design and Setting}

A community based cross sectional survey study was designed to determine the current malaria prevalence and its associated factors in and around Arjo Didhessa sugar factory, Western, Ethiopia. Additionally a retrospective 5-year (2013-2017) malaria trend analysis was designed at Arjo-Dhidhessa health centre to determine malaria cases. The health center provides a general health service in addition to malaria control and treatment for the catchment population. In and around Arjo Didhessa sugar cane plantation area, five study clusters (Abote Didhessa, Command 2, Command 5, Command 8 and main camp) were purposefully selected and used for survey study. Map of the study area is found in annex (Figure 1).

Study area and population 
The cross sectional survey study was conducted in five study clustersfound in and around Arjo Didhessa sugar factory from May-September 2017. Four study clustersfound in the sugar factory and the remaining one surrounding the factory. The area is located at Western Ethiopia of Oromiya Regional State in eastern Wollega, Eilu Ababora and Jimma Zones at the Didhessa Valley at a distance of 540 kilo meters from the capital through the route of Addis Ababa-Jimma-Beddele-Nekemet Road. The study area has generally a lowland climate with an altitudinal rage of 1570-1275 masl. The mean annual rainfall is 801-1400 mm. Maize, tef (Eragrostis teff) and pepper are produced for food and income. A small-scale animal husbandry is also practiced. The Factory in total has 20,000 hectares of land cultivated with cane. The factory has 800 permanent and 1,000 temporary workers. Sixty four residential houses and two service giving buildings with one health center were constructed and functional. This inevitably leads to attraction of more labor force to this irrigated area. In the present survey study, a total of 452 study subjects were included, individuals of both sexes and age 5 years.

Calculation of sample size $(n)$ was done using the formula for estimating single proportion $\left(n=Z^{2} P(1-P)\right.$ $/ d 2$ ), Where $n=$ sample size $d=$ worst accepted value/marginal error, $Z=$ is statistic value for level of $95 \%$ confidence, is 1. 96; $\mathrm{P}=$ is expected prevalence or proportion which is 0.5 . However, since there were no previous or pilot malaria studies conducted in the area and data from the clinic were studied only after the epidemiological study was done, for the survey study $50 \%$ was assumed for prevalence (P). A minimum of 384 samples ( $n$ ) was generated using $5 \%$ marginal error (d) as shown below. Therefore, once the minimum number of sample was obtained, to get the largest sample size $17 \%$ contingency was added and 452 study subjects were enrolled, in which this sample size refers to the survey study only.

All randomly selected household heads and family members in the selected study clusters of Arjo Didhessa sugar cane plantation area were the source of the study population for the interview questionnaire and parasitological blood film investigation, respectively. A pre-coded questionnaire was administered to all family heads living in the selected households in a face- to- face interview approach. In addition, blood smears were collected from a finger of all members in the selected households for smear test. All household heads and family members who were available in the selected households during sample collection were eligible for interview and smear test respectively. However, relatives who came during the study period and family members who were not available in the home were excluded.

Data collection Procedures: three data sources were used.Malaria parasite microscopy All 452 individuals were requested to give micro blood sample (capillary blood from fingertip) of blood by the end of interview for parasitological examination. Two separate blood films; thick and thin were made on frosted glass slide for each individuals by a medical laboratory technician according to the standard operating procedure (SOP) protocol and standards [11].Slides were labeled and air-dried horizontally in a slide tray, and thin films were fixed with methanol after drying. Slides were stained with $3 \%$ Giemsa for 30-45 minutes at Arjo Didhessa sugar factory health center laboratory unit. Blood slides were read by 100X objective lens of Olympus microscope. A minimum of 200 fields were scanned to report negative slides.After cross-checking, the slides were reported as either negative for blood parasites, $P$. falciparum positive, $P$. vivax positive or mixed infection with both $P$. falciparum and $P$. vivax. The staining technique 
and blood film examination was conducted according to a standard of WHO Protocols [11]. All slides were cross checked blindly by independent microscopist and concordant results were reported as a final result.

\section{Structured Questionnaire Survey}

For the cross sectional survey study a structured questionnaire addressing socio-demographics, household characteristics and health behavioral factors and other duty category of the residents were used. The survey questionnaires were based on the malaria indicator survey household questionnaires, which were filled by the participants. Before going to the actual data collection, pretest of the questionnaire was done among $5 \%$ of the sample in nearby localities not involved in the actual data collection. A total of 10 data collectors, who had previous experience with malaria surveys, and 2 supervisors were involved after two days of intensive training. Moreover, the investigators were involved in the provision of training for data collectors and monitoring the overall data collection activities. The questionnaire was administered to 452 volunteers by trained interviewers considering to the time schedule of the participants.

Retrospective health facility data In Arjo Didhessa sugar factory health center, peripheral blood is routinely examined for malaria parasite detection according to the standard operating procedure of malaria in Ethiopia. We retrieved Data of malaria in the past 5-year (2013-2017) from the health service laboratory unit registry to compute the trend of malaria in the community. We extracted specific data on species of malaria identified, total cases suspected of malaria, annual, monthly and seasonal cases of malaria.

\section{Data entry and analysis}

Data were checked for completeness and consistency, and entered (twice) into statistical program for social sciences version 20.0 for Windows (SPSS Inc, Chicago,IL, USA). Descriptive analysis was computed for both dependent and in dependent variables. The frequency distribution of both dependent and independent variables were worked out and the association between the independent and dependent variables were measured and tested using OR and $95 \% \mathrm{Cl}$. We used binary logistic regression to build the fitting model for multivariate analysis. We selected the candidate variables for multivariate analysis based on purposeful selection of the variables at $P=0.25$ in the univariate analysis. The significant level was considered at $\mathrm{P}<0.05$ in the multivariate model.Odds ratio $(\mathrm{OR})$ at $95 \%$ confidence interval was considered to see association between the prevalence of malaria and the independent variables. Prevalence of malaria is considered as the main outcome variable in the analysis. All variables with a crude odds ratio having a p-value less than 0.2 were transferred to the final adjusted model. Accordingly, three variables were found to become predictors of malaria prevalence among the surveyed individuals. Individuals who practiced or those who have had an experience of outdoor sleeping during night time were seventy seven times more likely to acquire malaria when compared to indoor sleeping counterparts (AOR, 77 (8-789). Bed net utilization behavior and indoor chemical spray were among the variables negatively associated with existing malaria prevalence in the study area. Among the surveyed groups those individuals who do not utilize bed nets in their home have eleven times odds of developing malaria 
(AOR, 11(2-65) than those who used bed net effectively. In the meanwhile, those respondents who were absent during spray time were or those who didn't spray their home have fourteen times odds to develop malaria than who have sprayed their home (AOR, 14(1.3-158) (Table 3).

\section{Results}

A community based cross sectional survey study was undertaken with a total of 452 study participants, in which majority of them (67.9\%) were male with mean age and SD of 26.5 and 12 respectively. Around $46 \%$ of the respondents were daily laborers and about $73 \%$ have no formal educational background or were educated up to primary cycle in concern to educational attainment. Regarding their marital status, half of the surveyed residents were married and the remaining portion was single. A significant (60\%) of the respondents were dwelling in a conventional type housing unit. Majority $(70 \%)$ of the total respondents lived in the surrounding area for at least five years or more. Furthermore, the study site was divided into five clustering segments which contributed a comparable amount of study participants of which the highest number $132(30 \%)$ was chosen from 'Abote Didhessa' clustering unit (Table 1).

Among the total (452) of surveyed participants a blood sample was received from 443(98\%) assented individuals for parasitological examination and a total of 14 laboratory confirmed malaria parasites were found to exist giving an overall malaria prevalence of $3.1 \%$ around the sugar factory during the study period. Like the other remaining parts of Ethiopia only the two major species of malaria; Plasmodium vivax 8 (57\%) and Plasmodium falciparum 6 (43\%) were detected during the survey. No significant difference was observed regarding malaria distribution among the four clustered communities but more number of malaria parasites was detected in blood samples of respondents from Abote Didhessa and all individuals with malaria case were living in a conventional housing type unit (Table 2).

A binary logistic regression model was used to identify factors associated with malaria prevalence in the vicinity. In bivariate model all variables were included to identify candidate variables fitting to the final model of multivariate analysis.

Among the surveyed individuals, three variables were found to become predictors of malaria prevalence. Individuals who practiced an experience of outdoor sleeping were seventy seven times more likely to acquire malaria when compared to indoor sleeping counterparts (AOR, 77 (8-789). In the study area indoor chemical spray and bed net utilization behavior were among the variables negatively associated with existing malaria prevalence. Among the surveyed groups those individuals who do not utilize bed nets in their home have eleven times odds of developing malaria (AOR, 11(2-65) than those who used bed net effectively. On the other hand, those individuals who were absent during spray time have fourteen times odds to develop malaria than who have sprayed their home (AOR, 14(1.3-158) (Table 3).

Regarding retrospective study of annual trends of malaria cases, within the last five successive years (2013-2017), in Arjo Didhessa sugar cane plantation area a total of 65,275 patients were visited the prime public health center found around the sugar factory. Among these patients, there was a total of 
4,164 laboratory confirmed malaria cases which yields an estimated malaria case proportion of $6.38 \%$ and mean annual malaria cases of 832.8.

During the last five years (2013-2017) the retrospective clinical data revealed a slight fluctuating trend of malaria occurrence. The peak malaria case occurrence was in $2013(13.84 \%)$ and less malaria occurring year was in 2017 (1.24\%) showing remarkable reduction in 2017.In general, the trend of malaria observed over the five years exhibited peak incidence in the year 2013 and declining in 2014 and 2015 with steady fall in 2016 and 2017. Malaria trend analysis was not described for 'Abote Didhessa' clustering unit, which is attributed to lack of consecutive retrospective clinical data. The dominant types of plasmodium species was Plasmodium vivax 3,170(4.85) over the five years (Table 4).

Even though there was reduction of malaria cases, currently malaria cases were occurred in every month of a year with mean monthly cases of 347 and the peak cases of malaria was depicted in the month of August (732 cases) which showed the highest monthly prevalence of $1.12 \%$. With regard to the identified plasmodium species, both species of plasmodiumwere reported in each year with Plasmodium vivax being the predominant species used to be reported in the study area. Plasmodium vivax accounted for 76.12\% and Plasmodium falciparum $18.63 \%$ mixed (both Plasmodium falciparum and Plasmodium vivax) $5.23 \%$ of the total malaria cases. Both species of malaria was seemingly decreasing uniformly every year and no fluctuation and trend shift was observed.

\section{Discussion}

In this study the investigators tried to assess overall impact of malaria as a public health problem in and around Arjo Didhessa sugar cane plantation areas. Malaria prevalence and malaria risk factors were assessed using community based cross sectional study design and also malaria trend analysis was depicted in the area five years prior to the study.

Among a total of 452 study participants, blood sample was received from 443 in which, the overall active malaria prevalence yielded during the survey was 14 (3.1\%). Of the total malaria parasite detected Plasmodium vivax accounts 8 (57\%) and the remaining type 6 (43\%) is Plasmodium falciparum. The community prevalence of malaria explored in the current study is comparably similar with the finding of the study conducted in Fincha sugar factory, located at Western Ethiopia which reported overall malaria prevalence of $2.6 \%$ [12]. Similarly, the study is in line with the finding of other study carried out in central part of Ethiopia that reported $4.2 \%$ [13]. The present study is also in agreement with previous study in Gedo Zone, Sothern Ethiopia, in which the identified dominant species is Plasmodium vivax followed by Plasmodium falciparum, but in contrast with the overall prevalence (16\%) [14]. In the present finding there was no mixed infection reported which is comparable with study done in Metama Hospital in which the mixed infection is only $0.3 \%$ [15].The finding of the current study was also in concurrence with other study done in Sudan which reported 3.8\% prevalence of concomitant malaria [16].

According to 2015 national malaria indicator survey, the study area falls among highland fringes and moderate transmission risk woredas of Ethiopia with API (Annual Parasite Index) of 0-5 [2]. This result is 
much higher than the result of national malaria indicator survey which is $0.55 \%$. This disparity could be due to fact that, the study area apart from its geographical location in malarious areas is a newly established plantation site and presence of irrigations which makes prone the area to a relatively higher percentage of malaria prevalence and also the survey was held at peak time of malaria transmission period.

Regarding to risk factors, individuals who practiced or those who have had an experience of outdoor sleeping during night time were seventy seven times more likely to acquire malaria when compared to indoor sleeping counterparts (AOR, 77 (8-789). Furthermore, bed net utilization behavior and indoor chemical spray were among the variables negatively associated with existing malaria prevalence in the study area. Among the surveyed groups those individuals who do not utilize bed nets in their home were eleven times more likely to develop malaria (AOR, 11(2-65) than those who used bed net effectively. This is in line with previous study in which outdoor sleeping, and bed net utilization were associated with the risk of malaria $(P<0.05)$ West Armachiho District, Northwest Ethiopia [17].

In the present study, the trend of malaria indicated that the peak cases was observed in the 2013 and cases reduction in 2014 and 2015 and steady fall in 2016 and 2017. This is in agreement with seven-year retrospective study from Metema Hospital, Northwest Ethiopia in which positive rate of malaria within the last seven years (2006-2012) was almost constant with slight fluctuation [15]. Even though the trend of malaria is moving down, the prevalence of malaria observed in the community is still higher than the yearly malaria case proportion seen at the clinic last year. This rise is owing to period of the data collection being the major malaria transmission period.

\section{Limitations Of The Study}

Although the investigators tried to assess a relatively wide range of malaria situation, focusing on current status supplemented with cumulative prevalence in the past, the study was not rid of limitations. The main limitation of the study was incompleteness and inconsistency of retrospective data used to analyze trend prevalence of malaria. In the retrospective part of the study, only the total malaria cases over the five years were presented not the prevalence of malaria.

The diagnosis of malaria was used to be reported by microscopy in the health center during the past five years prior to the study. If one method missing or malfunctioning, the other was used as an alternative method. Hence, for our consumption we reported the data arising from combination of both techniques of diagnosis. While calculating the trend analyses some important variables like age, sex and residence were not included because of the incompleteness of the data in some specific years.

\section{Conclusions}

Even though promising and progressive changes have been made over the few past years around the area in prevention and control of malaria, the prevalence of malaria observed in the area $3.1 \%$ is still 
higher as compared to national prevalence of malaria. The area is potentially at risk for high transmission of malaria since there is large scale irrigational dams and canals that sets optimum growth requirement for mosquito vector. Therefore, we recommend further strengthening of malaria prevention and control strategies. Additionally, educative training and awareness creation opportunities should be provided for workers in the plantation area on malaria prevention.

\section{Abbreviations}

AIDS Acquired Immune Deficiency SyndromeAOR Adjusted Odds RatioCOR Crude Odds RatioGPS Global Positioning SystemHIV Human Immune Deficiency virus IPT Intermittent Preventive Treatment IRS Indoor Residual SprayingITN Insecticide Treated Net IVM Integrated Vector Management SOP Standard Operating Procedure TB Tuberculosis WHO World Health Organization

\section{Declarations}

\section{Ethics and consent to participate}

Ethical clearance was obtained from Research ethics review committee of Wollega University. A collaborative linkage letter informing the respective health facility administrators were written by Wollega University and permission obtained. The aim of the study was expounded to the participants and malaria suspected cases were involved in the survey study after obtaining informed consent. Assent was obtained from guardians for children under age of 18. The informed consent obtained was in written using their local language. During the sample collection we remained adherent to aseptic techniques. Any personal identifier was delinked to the raw data before the final analysis. All study subjects found positive for malaria cases were treated without any payment at Dhidhessa health center under thesupervision of health care providers according to the national protocol.

Consent for publicationNot applicableAvailability of data and material

The data set generated from patients' clinical record is not publicly available to protect patient confidentiality. Unidentifiable data can be obtained from the corresponding author upon reasonable request.

Competing interestsThe authors declare that they have no competing interests regarding publication of this manuscriptFunding This work was funded by the office of vice president for research and technology transfer, Wollega University.

\section{Authors' contributions}

MD contributed to conception and design, acquisition of data. MD, RD and GT substantially contributed to conception and design of the study, analysis and interpretation of data, drafting the manuscript and 
revisiting it critically for important intellectual content. RD and GT contributed to microscopic examination and all authors approved the final version.

\section{Acknowledgements}

The authors are grateful for study participants involved in the study. Authors also thank data collectors and laboratory technologists for their strong commitment during data collection. Also our special thanks go to Arjo Didhessa sugar factory administrative staffs in general and Health Centre administrative staffs in particular for their essential support during site survey. This work was supported by the office of vice president for research and technology transfer, Wollega University for which we are thankful.

\section{References}

1. World Health Organization: World malaria report 2017. Geneva, Licence: CC BY-NC-SA 3.0 IGO.

2. Federal Ministry of health and Ethiopian public health institute: Ethiopian national malaria indicator surveys 2015, Addis Ababa Ethiopia.

3. WHO. World malaria report. Geneva, Switzerland: World Health Organization; 2018.http://www. Who.int/malaria.

4. EMIS. Ethiopian national malaria indicator survey. Addis Ababa, Ethiopian Public Health Institute.2016.

5. Woyessa A, Deressa W, Ali A, Lindtjørn B: Prevalence of malaria infection in Butajira area, southcentral Ethiopia. Malaria journal 2012, 11(1): 84.

6. Ethiopian Public Health Institute, Federal Ministry Of Health, National Malaria Control Team, World Health Organization, Addis Ababa University, The INFORM Project, Department of Public Health Research and Kenya Medical Research Institute - Wellcome Trust Progamme: An Epidemiological Profile of Malaria In Ethiopia, 2014, Nairobi, Kenya Version 1.0.

7. Woyessa A, Deressa W, Ali A, Lindtjørn B: Malaria risk factors in Butajira area, south-central Ethiopia: a multilevel analysis. Malaria journal 2013, 12(1): 273.

8. FMoH: Malaria diagnosis and treatment guidelines for health workers in Ethiopia. 2nd ed. Federal Democratic Republic of Ethiopia Ministry of Health 2004, Addis Ababa. 58 pp.

9. Feleke DG, Gebretsadik D, Gebreweld A: Analysis of the trend of malaria prevalence in Ataye, North Shoa, Ethiopia between 2013 and 2017. Malaria journal 2018, 17(1): 323.

10. Gemechu T, Samuel A, Yewhalaw D: Ten Years Trend Analysis of Malaria Prevalence and its Correlation with Climatic Variables in Sibu Sire District, East Wollega Zone, Oromia Regional state, Western Ethiopia: A Retrospective Study. Sci.Technol.Arts Res J.2015, 4(4): 99-105.

11. WHO (1993): Basic Laboratory Methods in Medical Parasitology.World Health Organization, Geneva.

12. Chala B, Petros B: Malaria in Finchaa sugar factory area in western Ethiopia: assessment of malaria as public health problem in Finchaa sugar factory based on clinical records and parasitological 
surveys, western Ethiopia. J. Parasitol. Vector Biol. 2011, 3(4): 52-58.

13. Tesfaye S, Belyhun Y, Teklu T, Mengesha T, Petros B: Malaria prevalence pattern observed in the highland fringe of Butajira, Southern Ethiopia: a longitudinal study from parasitological and entomological survey. Malaria journal 2011, 10(1): 153.

14. Molla E, Ayele B: Prevalence of Malaria and Associated Factors in Dilla Town and the Surrounding Rural Areas, Gedeo Zone, Southern Ethiopia. J Bacteriol Parasitol 2015: 6:5

15. Ferede G, Worku A, Getaneh A, Ahmed A, Haile T, Abdu Y, Tessema B, Wondimeneh Y, Alemu A: Prevalence of malaria from blood smears examination: a seven-year retrospective study from Metema Hospital, Northwest Ethiopia. Malaria research and treatment 2013, Article ID 704730, 5 pages.

16. van den Bogaart E, Berkhout MM, Nour AB, Mens PF, Talha ABA, Adams ER, Erika van den Bogaart, Marieke MZ, Berkhout, Ayman BYM Nour, Pètra F Mens, Al-Badawi A Talha, Emily R Adams, Ahmed HBM, Abdelrahman SH, Ritmeijer K,Bakri YM, Schallig HD: Concomitant malaria among visceral leishmaniasis in-patients from Gedarif and Sennar States, Sudan: a retrospective case-control study. BMC public health 2013, 13(1): 332.

17. Aschale Y, Mengist A, Bitew A, Kassie B, Talie A: A Prevalence of malaria and associated risk factors among asymptomatic migrant laborers in West Armachiho District, Northwest Ethiopia. Research and Reports in Tropical Medicine 2018, 9: 95-101.

\section{Tables}

Table 1 Characteristics of Sociodemographic, socioeconomic and housing condition of study participants living in and around Arjo Didhessa sugar cane plantation area (MayNovember, 2017). 


\begin{tabular}{|c|c|c|}
\hline Variables $(\mathrm{n}=452)$ & Category & Frequency n (\%) \\
\hline \multirow[t]{3}{*}{ Sex } & Male & $307(67.9 \%)$ \\
\hline & Female & 145(32.1\%) \\
\hline & Age less than 15 years & $32(7.1 \%)$ \\
\hline \multirow[t]{3}{*}{ Age } & Age $15-30$ years & $313(69.2 \%)$ \\
\hline & Age greater than 30 years & 107(23.7\%) \\
\hline & Daily laborer & $208(46.0 \%)$ \\
\hline \multirow[t]{4}{*}{ Occupation } & Farmer & $117(25.9 \%)$ \\
\hline & Government employee & $83(18.4 \%)$ \\
\hline & Others* $^{*}$ & $44(9.7 \%)$ \\
\hline & No formal education/read \&write only & $166(36.7 \%)$ \\
\hline \multirow[t]{3}{*}{ Educational Status } & Primary education & $165(36.5 \%)$ \\
\hline & Secondary education & $70(15.5 \%)$ \\
\hline & Tertiary education & $51(11.3 \%)$ \\
\hline \multirow[t]{2}{*}{ Marital Status } & Single & $234(51.8 \%)$ \\
\hline & Married & $218(48.2 \%)$ \\
\hline \multirow[t]{2}{*}{ Duration In The Village } & Stay $<=$ five years & $316(69.9 \%)$ \\
\hline & Stay more than 5 years & $136(30.1 \%)$ \\
\hline \multirow[t]{5}{*}{ Study clusters } & Abote Didhessa & $132(29.2 \%)$ \\
\hline & Command 2 & $74(16.4 \%)$ \\
\hline & Command 5 & $90(19.9 \%)$ \\
\hline & Command 8 & $64(14.2 \%)$ \\
\hline & Main camp & $92(20.4 \%)$ \\
\hline \multirow[t]{3}{*}{ Housing Unit } & Conventional & $268(59.3 \%)$ \\
\hline & Improved & $42(9.3 \%)$ \\
\hline & Others $^{* *}$ & $142(31.4 \%)$ \\
\hline
\end{tabular}

Keys

* Students, housewives, merchants

** Housing units made walls of iron bars,

Table 2 Prevalence of malaria among study participants living in and around Arjo Didhessa sugar cane plantation area (May-November, 2017) 


\begin{tabular}{|c|c|c|c|c|}
\hline $\begin{array}{l}\text { Variables } \\
(n=452)\end{array}$ & Category & $\begin{array}{l}\text { Number of } \\
\text { surveyed } \\
\text { participants }\end{array}$ & $\begin{array}{l}\text { Number of malaria parasite } \\
\text { detected per category }\end{array}$ & $\begin{array}{l}\text { Prevalence of malaria } \\
\text { per category (\%) }\end{array}$ \\
\hline \multirow[t]{3}{*}{ Sex } & Male & 307 & 13 & $4.23 \%$ \\
\hline & Female & 145 & 1 & $0.68 \%$ \\
\hline & Age less than & 313 & 10 & $3.2 \%$ \\
\hline \multirow[t]{4}{*}{ Age } & 15 years & & & \\
\hline & $\begin{array}{ll}\text { Age } & 15-30 \\
\text { years } & \end{array}$ & 107 & 2 & $1.87 \%$ \\
\hline & $\begin{array}{l}\text { Age greater } \\
\text { than } 30 \text { years }\end{array}$ & 32 & 2 & $6.25 \%$ \\
\hline & Daily laborer & 208 & 5 & $2.40 \%$ \\
\hline \multirow[t]{3}{*}{ Occupation } & Farmer & 117 & 4 & $3.42 \%$ \\
\hline & $\begin{array}{l}\text { Government } \\
\text { employee }\end{array}$ & 83 & 3 & $3.61 \%$ \\
\hline & Others* & 44 & 2 & $4.55 \%$ \\
\hline \multirow{2}{*}{$\begin{array}{l}\text { Marital } \\
\text { Status }\end{array}$} & Single & 234 & 8 & $3.42 \%$ \\
\hline & Married & 218 & 6 & $2.75 \%$ \\
\hline \multirow[t]{2}{*}{$\begin{array}{l}\text { Duration In } \\
\text { The Village }\end{array}$} & $\begin{array}{l}\text { Stay }<=\text { five } \\
\text { years }\end{array}$ & 316 & 8 & $2.53 \%$ \\
\hline & $\begin{array}{l}\text { Stay more } \\
\text { than } 5 \text { years }\end{array}$ & 136 & 6 & $4.41 \%$ \\
\hline Study & Abote & 132 & 6 & $4.5 \%$ \\
\hline \multirow[t]{6}{*}{ Clusters } & Didhessa & & & \\
\hline & Command 2 & 74 & 3 & $4.05 \%$ \\
\hline & Command 5 & 90 & 1 & $1.1 \%$ \\
\hline & Command 8 & 64 & 1 & $1.56 \%$ \\
\hline & Main camp & 92 & 3 & $3.26 \%$ \\
\hline & Conventional & 268 & 14 & $5.2 \%$ \\
\hline \multirow[t]{2}{*}{ Housing Unit } & Improved & 42 & 0 & $0 \%$ \\
\hline & Others & 142 & 0 & $0 \%$ \\
\hline \multirow[t]{3}{*}{$\begin{array}{l}\text { Parasite } \\
\text { species }\end{array}$} & $\begin{array}{l}\text { Plasmodium } \\
\text { vivax }\end{array}$ & 452 & 8 & $1.8 \%$ \\
\hline & $\begin{array}{l}\text { Plasmodium } \\
\text { falciparum }\end{array}$ & 452 & 6 & $1.3 \%$ \\
\hline & $\begin{array}{l}\text { Total malaria } \\
\text { parasite }\end{array}$ & 452 & 14 & $3.1 \% * *$ \\
\hline
\end{tabular}

Key *Students, housewives, merchants

** Overall prevalence of malaria 
Table 3 Factors associated with malaria prevalence among study participants living in and around Arjo Didhessa sugar cane plantation area (May-November, 2017).

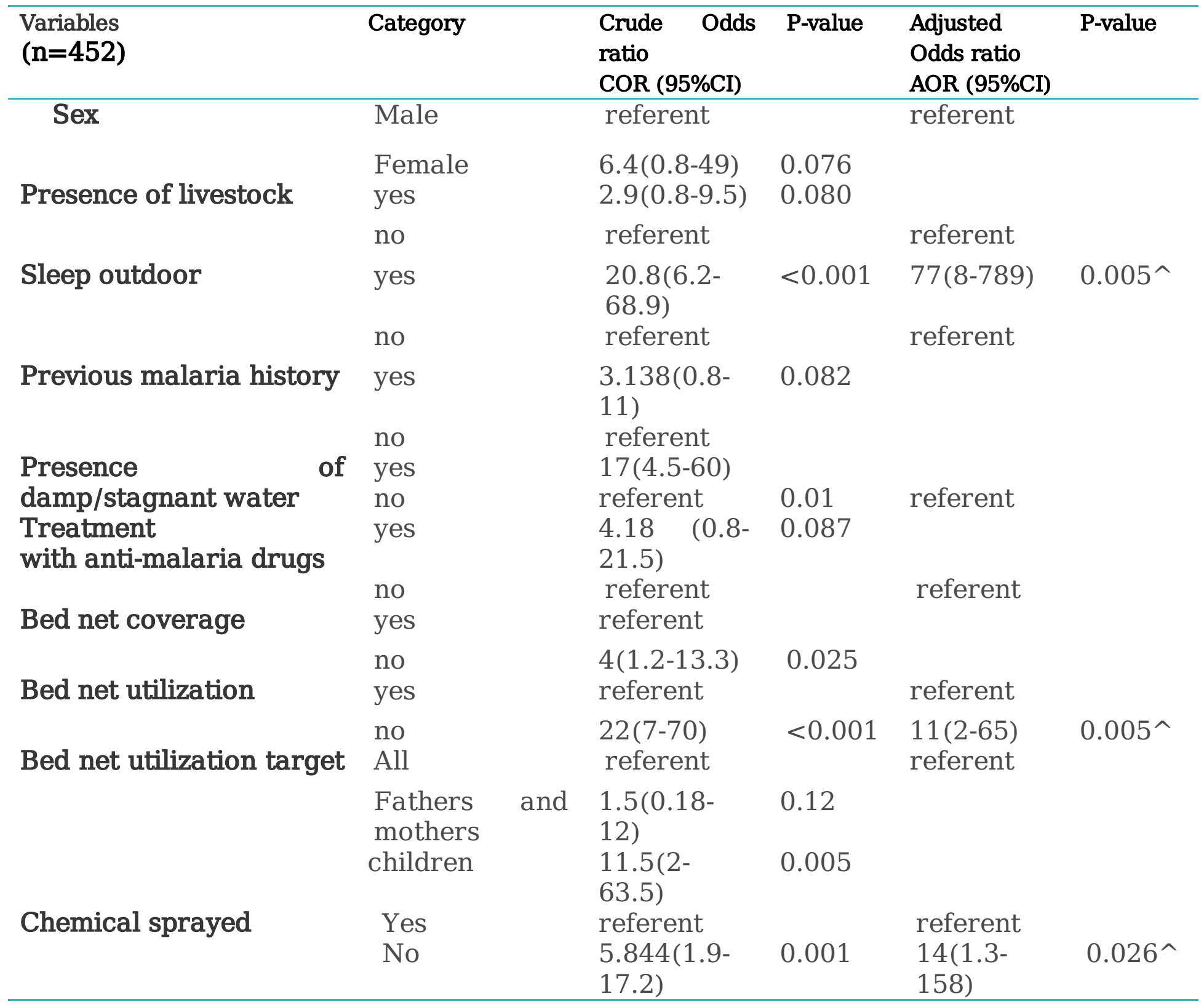

$\mathrm{Key}^{\wedge}$ statistically significant variables at p-value $=0.05<0.05$ 
Table 4 Annual malaria trend case proportion in and around Arjo Didhessa sugar cane plantation area (2013-2017)

\begin{tabular}{llllll}
\hline Year(E.C) & Total OPD & \multicolumn{4}{c}{ Case proportion No (\%) } \\
\cline { 3 - 6 } 2013 & 4234 & Pf & Pv & Mixed & Total \\
2014 & 11234 & $260(2.31)$ & $1081(9.62)$ & $61(0.54)$ & $1402(12.48)$ \\
2015 & 15318 & $200(1.31)$ & $1317(8.6)$ & $100(0.65)$ & $1617(10.56)$ \\
2016 & 16928 & $44(0.26)$ & $287(1.7)$ & $10(0.06)$ & $341(2.01)$ \\
2017 & 17561 & $37(0.21)$ & $178(1.012)$ & $3(0.02)$ & $218(1.24)$ \\
total & 65275 & $776(1.19)$ & $3170(4.85)$ & $218(0.33)$ & $4164(6.38)$ \\
\hline
\end{tabular}

Figures 


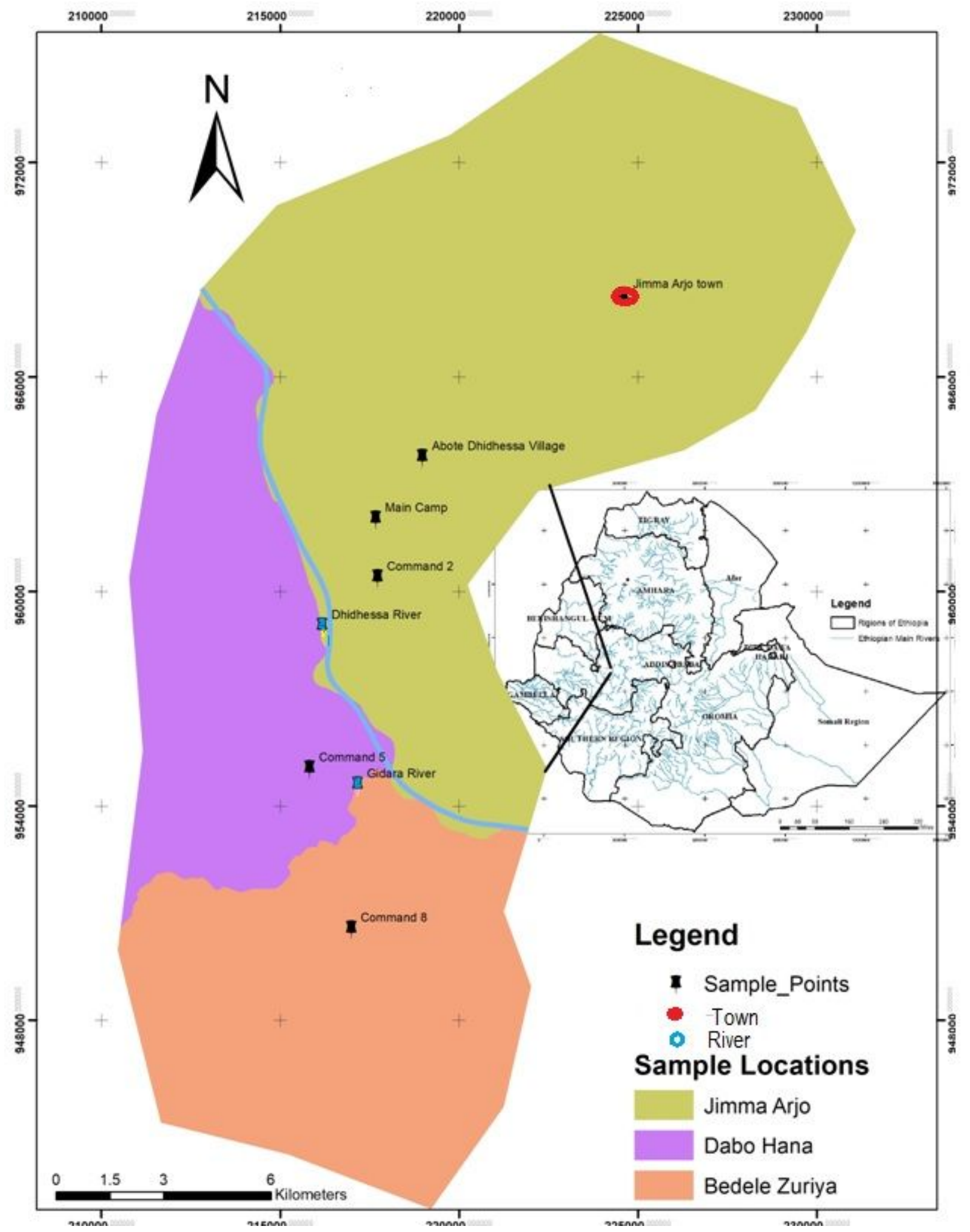

Figure 1

Location of the study sites, Arjo Didhessa sugar cane plantation area, Western Ethiopia (Source: Garmin 72 GPS) 


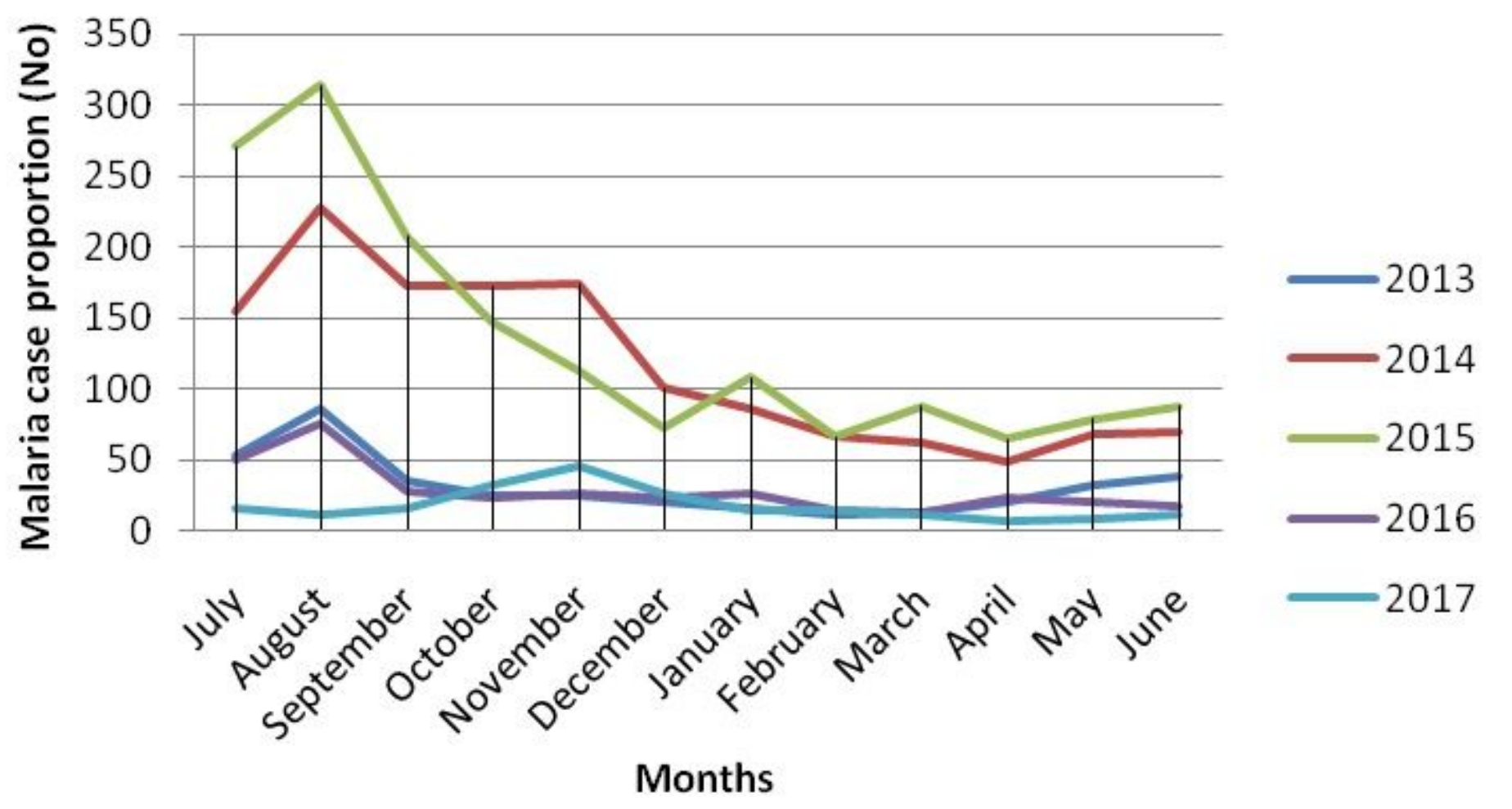

\section{Figure 2}

Monthly and yearly malaria trend case proportion in and around Arjo Didhessa sugar cane plantation area (2013-2017)

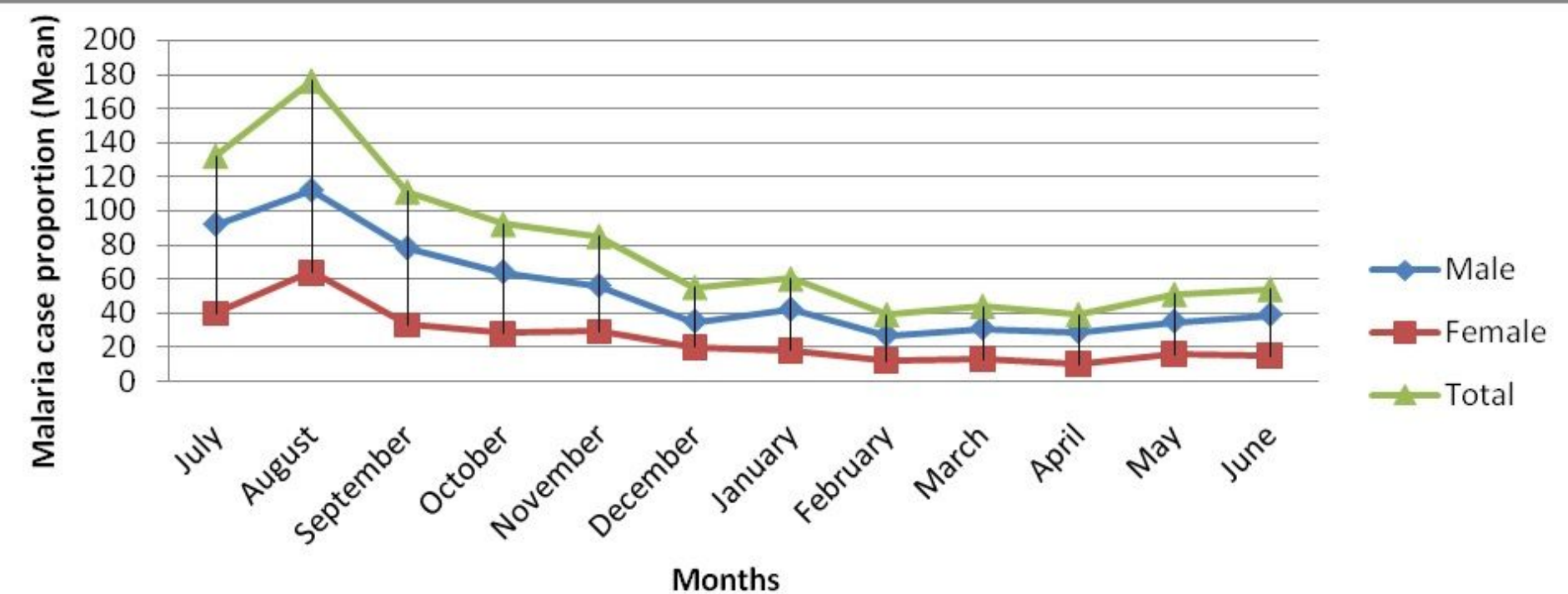

Figure 3

Mean monthly malaria trend case proportion by sex in and around Arjo Didhessa sugar cane plantation area (2013-2017) 